\title{
Funcionalidade em sujeitos com transtorno depressivo maior: avaliação das propriedades psicométricas da escala Functioning Assessment Short Test (FAST) em amostra brasileira
}

\author{
Functionality in subjects with major depressive disorder: \\ evaluation of psychometric properties of Functioning \\ Assessment Short Test (FAST) scale in Brazilian sample \\ Juliana de Almeida Prado ${ }^{1}$ \\ https://orid.org/0000-0003-2485-9111 \\ Giovanni Gurgel Aciole ${ }^{1}$ \\ hittp:///orid.org/0000-0002-6400-8293 \\ Jair Lício Ferreira Santos ${ }^{2}$ \\ hitps://lorid. org/000-0001-7367-4418
}

\section{Palavras-chave}

Funcionalidade, depressão, propriedades psicométricas, escala.

\section{RESUMO}

Objetivos: $O$ transtorno depressivo maior (TDM) é muito prevalente e incapacitante, sendo comum a persistência de prejuízos funcionais após a remissão clínica. Pesquisas que se proponham a estudar a reabilitação em TDM são necessárias. Há carência de instrumentos que avaliem a funcionalidade em sujeitos com TDM. Objetiva-se observar as propriedades psicométricas da escala FAST em amostra brasileira, casos $(n=44)$ e não casos $(n=44)$ de TDM. Métodos: a) Questionários com informações socioeconômicas e escala Mini International Neuropsychiatric Interview 5.0.0 para rastrear em sala de espera de serviços ambulatoriais de duas universidades públicas do Estado de São Paulo; b) entrevistas face a face com os instrumentos FAST, Avaliação Global de Funcionamento e Escala de Hamilton para Depressão. Resultados: Houve fortes correlações entre FAST e AGF (rho =-0,85) e entre cada subescala e a escala total (rho =0,86), boa consistência interna (alfa de Cronbach 0,98) e confiabilidade teste-reteste (Kappa ponderado >0,84). O estudo de validade discriminativa evidenciou que a FAST discrimina os casos de não casos de TDM, assim como moderados/graves dos demais. Conclusão: As propriedades psicométricas da FAST em 88 sujeitos mostraram boa confiabilidade e validade para aferir o impacto da depressão na funcionalidade em brasileiros com TDM. Recomendam-se estudos futuros com amostras maiores.

\section{ABSTRACT}

Objectives: Major depressive disorder (MDD) is very prevalent and incapacitating and there is a common persistence of functional impairment after clinical remission. Research that proposes to study rehabilitation in TDM is necessary. There is a lack of instruments that evaluate

1 Universidade Federal de São Carlos (UFSCar), Departamento de Medicina, São Carlos, SP, Brasil. 2 Universidade de São Paulo (USP), Faculdade de Medicina de Ribeirão Preto, Ribeirão Preto, SP, Brasil.

Recebido em 3/Mar/2019

Aprovado em

24/Mar/2019

Endereço para correspondência: Juliana de Almeida Prado

Rodovia Washington Luiz, Km 235

13565-905 - São Carlos, SP, Brasil

E-mail: jjbrprado@gmail.com 


\section{Keywords}

Functionality, depression, psychometric properties, scale. the functionality in subjects with TDM. We aim to observe the psychometric properties of the FAST scale in Brazilian sample, cases $(n=44)$ and not cases $(n=44)$ of MDD. Methods: $a)$ Questionnaires with socioeconomic information and Mini International Neuropsychiatric Interview 5.0.0 scale for screening in outpatient waiting room of two public universities of the State of São Paulo; b) face-to-face interviews with the FAST, Global Functional Assessment and Hamilton Depression Scale instruments. Results: Strong correlations between FAST and AGF (rho $=-0.85$ ) and between each subscale and total scale (rho =0.86), good internal consistency (Cronbach's alpha 0.98) and test-retest reliability weighted $>0.84$ ). The discriminative validity study evidenced that the FAST discriminates the cases of non-cases of MDD, as well as moderate/severe cases of the others. Conclusion: The psychometric properties of FAST in 88 subjects showed good reliability and validity to assess the impact of depression on functionality in Brazilians with MDD. Future studies with larger samples are recommended.

\section{INTRODUÇÃO}

O transtorno depressivo maior (TDM) é uma condição de saúde muito comum nas populações gerais e de trabalhadores ${ }^{1-4}$. Existem dados consistentes na literatura que permitem afirmar que o TDM não é apenas comum, mas também altamente incapacitante e associado a grande impacto socioeconômico, sendo, entre as doenças não fatais, a principal causa de years lost days (YLD) no mundo ${ }^{5}$. No Brasil, a depressão é responsável por 3,5\% do total da carga global das doenças ${ }^{4}$. O TDM tem evolução recorrente e com alta persistência de sintomas residuais entre os episódios ${ }^{6}$, de modo que uma parcela considerável dos sujeitos acometidos convive com déficits de funcionamento, mesmo após o episódio agudo, aumentando ainda mais o ônus associado a essa condição ${ }^{7,8}$.

No universo do trabalho, os prejuízos funcionais associados à depressão repercutem sobre a funcionalidade de diferentes formas, como diminuição da produtividade, aumento da ocorrência de erros e dos riscos de acidentes, conflitos interpessoais e inabilidade para lidar com situações adversas ${ }^{9}$. De fato, não é difícil compreender a repercussão dos sintomas depressivos sobre a funcionalidade no trabalho. Se o trabalhador se apresenta desmotivado, indeciso, com dificuldade para tomar decisões, para lidar com situações novas ou para acumular diversidade de tarefas, certamente terá problemas no desempenho, independentemente da sua natureza.

Um levantamento realizado com uma amostra representativa de trabalhadores do Canadá observou prevalência de depressão nos últimos 12 meses de 4\%, e entre esses 79\% referiram que os sintomas depressivos impactavam a performance para o trabalho, com uma média de 32 dias de afastamento do trabalho nos últimos 12 meses $^{10}$. Lerner e Henke e Lawrence et al. observaram maiores índices de desemprego entre sujeitos depressivos ${ }^{9-11}$.

Mensurar a funcionalidade dos transtornos de humor é muito mais complexo do que estimar os dias perdidos de trabalho e os prejuízos por queda da produtividade. Embora a aferição da funcionalidade a partir de desfechos simples como absenteísmo seja muito relevante, ela não permite uma compreensão mais rica desse fenômeno e importantes domínios do funcionamento como os aspectos cognitivos, relações interpessoais e autonomia podem ser negligenciados $^{12}$. Os instrumentos heteroaplicados e multidimensionais para aferição da funcionalidade mais utilizados são escalas gerais, por exemplo, a Avaliação Global de Funcionamento

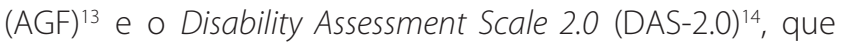
notoriamente falham em distinguir melhora clínica de recuperação funcional15. Da mesma forma, questionários autoaplicados também são problemáticos, em virtude de as respostas serem contaminadas pelo estado de ânimo dos sujeitos depressivos ${ }^{16}$. Assim, diante da escassez de métodos adequados, não são muitos os estudos que abordaram a funcionalidade associada ao TDM. Entre os realizados, grande parte utilizou instrumentos que não foram elaborados para dar conta das especificidades dos prejuízos associados à depressão, não contendo, portanto, informações clinicamente relevantes ${ }^{17}$.

Diante desse cenário, a Functioning Assessment Short Test (FAST) foi desenvolvida após os trabalhos da Sociedade Internacional de Transtorno Afetivo Bipolar, em Barcelona ${ }^{18}$, com o objetivo de suprir essas deficiências e ser uma escala de fácil aplicação, rápida e objetiva, capaz de avaliar as reais dificuldades no funcionamento psicossocial dos sujeitos com transtornos afetivos, permitindo, assim, seu uso em estudos internacionais e comparativos ${ }^{19,20}$. No Brasil, a FAST foi traduzida e validada em amostra de pacientes bipolares, por Cacilhas et al. ${ }^{17}$, e suas propriedades psicométricas apresentaram alta consistência interna (alfa de Cronbach > 0,9) e reprodutibilidade excelente após teste-reteste com coeficiente de Kappa de 0,90 $(p<0,01)^{16}$. Por não haver validação da FAST para sujeitos com TDM, este estudo se propôs a analisar as características psicométricas da escala em uma amostra brasileira de sujeitos depressivos. 


\section{MÉTODOS}

\section{Sujeitos}

Com o objetivo de avaliar as propriedades psicométricas da escala FAST em uma amostra de casos e não casos de TDM, foram selecionados, por conveniência, sujeitos com idades entre 18 e 65 anos, igualmente divididos entre homens e muIheres e pareados por idade e escolaridade. A busca ativa foi feita nas salas de espera dos serviços de saúde ocupacional e em ambulatórios de psiquiatria de duas universidades públicas do estado de São Paulo e em uma clínica psiquiátrica particular. Foram incluídos no grupo "casos" indivíduos com diagnóstico de TDM com base na aplicação da escala Mini International Neuropsychiatric Interview, versão 5.0.0 (MINI) ${ }^{21}$; foram excluídos aqueles com presença de quaisquer das comorbidades psiquiátricas constantes na MINI e, também, comorbidades clínicas que pudessem repercutir sobre a funcionalidade em geral, por exemplo, doenças neurológicas, ortopédicas e reumatológicas e cirurgias recentes. Todos os casos estavam em tratamento com antidepressivos em doses estáveis há pelo menos cinco semanas prévias à avaliação. Foram incluídos no grupo "não casos" de TDM aqueles com ausência de qualquer diagnóstico psiquiátrico com base na aplicação da escala MINI e excluídos, igualmente, os pacientes com presença de comorbidades clínicas que pudessem repercutir sobre a funcionalidade em geral. Todos os sujeitos inclusos neste estudo deveriam estar lúcidos e com boa capacidade de discernimento. A partir desses critérios, uma amostra de 88 sujeitos foi obtida, sendo 44 no grupo de casos e 44 no grupo de não casos.

\section{Materiais}

Os seguintes instrumentos foram utilizados neste estudo:

a. Functional Assessment Short Test (FAST): versão traduzida e adaptada para o Brasil por Cacilhas et al..17. É um instrumento heteroaplicado para avaliação objetiva e multidimensional da funcionalidade relativa aos últimos 15 dias. É composto por 24 itens, divididos em seis subescalas específicas para acessar áreas acometidas pelo TDM. A autonomia se refere à capacidade do sujeito de realizar ações sozinho ou tomar as próprias decisões; o funcionamento ocupacional se refere à capacidade do sujeito de se manter em um trabalho regular, ter uma performance estável e trabalhar em área compatível com sua capacitação e posição no trabalho; o funcionamento cognitivo diz respeito à capacidade do sujeito de se concentrar, fazer cálculos mentais simples, resolver problemas rotineiros, aprender uma nova informação e lembrar dessas informações aprendidas; habilidades financeiras envolvem a habilidade do sujeito de gerenciar suas finanças de maneira balanceada; o item "relacionamentos interpessoais" se refere à qualidade das relações de amizade e com familiares, à habilidade de participar de atividades sociais e relações sexuais e à habilidade para defender ideias e opiniões pessoais; atividades de lazer dizem respeito à performance em atividades físicas (esportes, exercícios) e ter atividades como hobbies ${ }^{18}$. O escore é determinado pela soma dos itens, que variam de 0 (indicando ausência de limitação) até 3 (indicando limitação severa). Está disponível nas versões em inglês, português e espanhol;

b. Avaliação Global de Funcionamento (AGF): instrumento mais utilizado para avaliação da funcionalidade, integrante do eixo $V$ do DSM-IV ${ }^{13}$. É dividida em 10 níveis de funcionamento com dois componentes cada (gravidade dos sintomas e funcionamentos psicológico, social e ocupacional), pontuados num continuum de 0 a $10^{13}$. Pontuações abaixo de 70 apontam para prejuízos na funcionalidade. Apesar de sua larga utilização, a AGF é um instrumento que não fornece informações específicas sobre quais áreas da funcionalidade estão acometidas, sendo pouco representativo dos reais impactos dos transtornos mentais;

c. Mini International Neuropsychiatric Interview (MINI): versão 5.0.0, traduzida e validada para o Brasil por Amorim ${ }^{21}$. É um instrumento utilizado para a elaboração de diagnósticos clínicos psiquiátricos baseados no DSM-IV, com questões dicotômicas (SIM/ NÃO) e composto por módulos, em um total de 19, que podem ser aplicados de forma independente ou combinada;

d. Escala de Hamilton para Depressão (HAM-D): versão de 21 pontos - padrão-ouro de referência para estudos de validação de outras escalas, foi desenvolvida para ser aplicada exclusivamente em sujeitos previamente diagnosticados com transtorno depressivo, com a finalidade de avaliar a gravidade dos sintomas, e não a sua existência ${ }^{22}$. Não se encontram na literatura pontos de corte determinados pelo autor da escala, aceitando-se, na prática clínica, a seguinte pontuação: de 0 a 7 pontos - eutímicos; de 8 a 13 pontos - depressão leve; de 14 a 18 pontos - depressão moderada; acima de 22 pontos - depressão gra$v^{23}$. Essa pontuação foi aplicada para categorização dos casos neste estudo;

e. Questionário de identificação: instrumento construído para o estudo em questão objetivando coletar dados relativos à caracterização socioeconômica e clínica da amostra.

\section{Procedimentos}

O estudo foi aprovado pelo Comitê de Ética em Pesquisa do Hospital das Clínicas da Faculdade de Medicina de Ribeirão Preto da Universidade de São Paulo (no 1.790.779). 
Os sujeitos participantes foram abordados nas salas de espera de uma clínica psiquiátrica e em serviços de saúde ocupacional e em ambulatório de psiquiatria de duas universidades públicas localizadas no estado de São Paulo. Foi assegurada a ausência de vinculação entre a concordância em participar do estudo e a concessão de benefícios previdenciários e/ou o seguimento no tratamento ambulatorial e/ ou de vantagens ou benefícios de qualquer natureza. Aqueles que concordaram em participar e que assinaram o termo de consentimento livre e esclarecido do estudo foram encaminhados para uma sala reservada, onde responderam, por meio de entrevista face a face, à aplicação da MINI. Posteriormente, os que preencheram os critérios para a inclusão nos grupos de casos e não casos foram convidados a prosseguir com a entrevista. Nessa mesma ocasião, foram então aplicados o questionário de identificação, a HAM-D, a FAST e a AGF. As entrevistas foram realizadas por entrevistador único, uma psiquiatra experiente e previamente treinada para a aplicação dos instrumentos de pesquisa.

\section{Análises estatísticas}

Os dados foram alocados em um banco de dados e submetidos a análises utilizando-se o Stata, versão $13^{24}$. As estatísticas descritivas das variáveis socioeconômicas, clínicas e da funcionalidade foram expressas como médias, medianas (intervalos interquartis) e desvios-padrão (DP) para as variáveis quantitativas e como frequência e percentagem para as qualitativas. A comparação dos grupos foi realizada a partir do teste de Kruskal-Wallis ${ }^{25}$.

As seguintes técnicas foram utilizadas para o estudo de validação da FAST:

1. Coeficiente de Spearman ${ }^{26}$, aplicado para as avaliações da validade concorrente entre escores totais da FAST e AGF e para a validade de construto a partir da correlação dos scores de cada subescala com o escore global da FAST. A magnitude das correlações encontradas foi classificada em 0 a 0,25: fraca; 0,26 a 0,50: moderada; 0,51 a 0,70: forte; acima de 0,71: muito forte ${ }^{27}$;

2. Área sob a curva ROC, com o intuito de observar a sensibilidade do instrumento em diferenciar os seguintes grupos: depressivos versus saudáveis e moderados/graves versus demais;

3. Coeficiente de Youden ${ }^{28}$ para estimar os pontos de corte na escala e diferenciar os seguintes grupos: depressivos versus saudáveis e moderados/graves versus demais;

4. Coeficiente Alpha de Cronbach, calculado para a escala total e cada uma das subescalas da FAST, para análise da consistência interna, conforme sugerido por Nunnally e Bernstein ${ }^{29}$;

5. Coeficiente Kappa ponderado ${ }^{30}$ para observar a confiabilidade teste-reteste. Foi aplicado em 38 sujeitos (19 homens e 19 mulheres) em duas ocasiões separadas por intervalos de 7 a $14 \operatorname{dias}^{30}$. A magnitude da concordância foi classificada a partir do critério de Landis e Koch ${ }^{31}$ - até 0,6: baixo; entre 0,6 e 0,8: moderado; acima de 0,8: muito bom.

Para todas as análises, adotou-se o nível de significância de $5 \%$.

\section{RESULTADOS}

\section{Caracterização sociodemográfica da amostra}

Compuseram a amostra total do estudo das propriedades psicométricas da escala FAST 88 sujeitos, sendo 44 casos e 44 não casos de TDM. Não foram registradas perdas, assim como qualquer dificuldade no preenchimento da escala, indicando boa aplicabilidade. A caracterização sociodemográfica e clínica da amostra está detalhada na tabela 1.

\section{Consistência interna}

Para a análise da consistência interna do instrumento, utilizou-se o alfa de Cronbach ${ }^{29}$, aplicado tanto para a escala total como para cada uma das seis subescalas. Os resultados foram bastante satisfatórios ao se observar um alfa de Cronbach de 0,98 para a escala como um todo, indicando que seus domínios são suficientemente homogêneos. Os índices observados para cada domínio foram os seguintes: Autonomia $(0,98)$, Trabalho $(0,96)$, Cognição $(0,97)$, Finanças $(0,96)$, Relações interpessoais $(0,94)$ e Lazer $(0,64)$.

Tabela 1. Caracterização sociodemográfica e clínica da amostra total incluída no estudo das propriedades psicométricas da escala FAST, segundo a presença ou ausência de TDM $(N=88)$

\begin{tabular}{|c|c|c|c|}
\hline Caracteristicas & $\begin{array}{l}\text { Sujeitos } \\
\text { depressivos } \\
(\mathrm{N}=44)\end{array}$ & $\begin{array}{l}\text { Controles saudáveis } \\
\qquad(\mathrm{N}=44)\end{array}$ & $\begin{array}{c}\text { Estatística (p } \\
\text { valores)\# }\end{array}$ \\
\hline Idade ${ }^{a}$ & $46,95(8,89)$ & $46,40(16,5)$ & $0,79^{*}$ \\
\hline \multicolumn{4}{|l|}{ Escolaridade ${ }^{b}$} \\
\hline Fundamental & $4(9,1)$ & $2(4,5)$ & \\
\hline Médio/técnico & $19(43,2)$ & $16(36,3)$ & $0,21^{* *}$ \\
\hline Superior completo & $21(47,7)$ & $18(40,9)$ & \\
\hline \multicolumn{4}{|l|}{ Sexob } \\
\hline Feminino & $21(47,7)$ & $23(52)$ & \\
\hline Masculino & $23(52,3)$ & $21(48)$ & $0,54^{* *}$ \\
\hline AGF $^{\circ}$ & $65(70-55)$ & $85(90-85)$ & $0,02 * * *$ \\
\hline Escore FAST Total' & $31(42-11)$ & $2,5(4-1)$ & $0,00^{3 * * * *}$ \\
\hline Escore FAST Autonomiac & $4(3-7)$ & $0(0-0)$ & $0,00 * * *$ \\
\hline Escore FAST Trabalhoc & $4(8-3)$ & $0(0-0)$ & $0,00^{* * *}$ \\
\hline Escore FAST Cogniçãoc & $6(7-3)$ & $0(0-0)$ & $0,00 * * *$ \\
\hline Escore FAST Finançasc & $2(4-2)$ & $0(0-0)$ & $0,00^{* * *}$ \\
\hline $\begin{array}{l}\text { Escore FAST Relações } \\
\text { Interpessoais }\end{array}$ & $9(12-7)$ & $0(1,75-0)$ & $0,00 * * *$ \\
\hline Escore FAST Lazerc & $3(5-2)$ & $1(2-0)$ & $0,00 * * *$ \\
\hline
\end{tabular}




\section{Confiabilidade teste-reteste}

Para aferir a confiabilidade teste-reteste, foram selecionados 38 sujeitos. A média de idade foi de 47,2 anos, sendo 50\% do sexo feminino. $O$ reteste foi aplicado de 7 a 14 dias após a primeira entrevista.

A avaliação da estabilidade da FAST foi observada a partir do coeficiente de confiabilidade Kappa ponderado, uma vez que a escala é composta por variáveis ordenadas ${ }^{30}$. A tabela 2 apresenta os resultados.

Observou-se elevada estabilidade, com valor de Kappa ponderado maior que 0,84 [IC 95\% (0,72-0,93)]. O domínio "Autonomia" foi o que se mostrou mais estável (Kappa ponderado $=1,0$ ) e o domínio "Relações interpessoais" foi o menos estável (Kappa ponderado $=0,86$ ), embora com resultados também excelentes.

\section{Validade concorrente}

A validade de critério concorrente foi aferida a partir da correlação com a versão em português da $A G F^{13}$ por meio do coeficiente de Spearman ${ }^{26}$. Os resultados da análise da correlação estão expressos na tabela 3.

Observa-se correlação negativa altamente significativa (rho $=-0,85, p<0,01$ ), indicando que sujeitos com boa funcionalidade aferida pela FAST obtiveram elevadas pontuações na AGF.

\section{Validade de construto convergente}

Todas as correlações entre as subescalas e o escore global da FAST foram significativas, conforme os dados apresentados na tabela 4.

Tabela 2. Valores obtidos e esperados na aplicação da FAST e correlações Kappa ponderado ( $N=38)$

\begin{tabular}{|c|c|c|c|c|c|}
\hline Item & Valores obtidos $(\%)$ & Valores esperados (\%) & Kappa ponderado & Desvio-padrão & IC (95\%) $)^{\mathrm{a}}$ \\
\hline \multicolumn{6}{|c|}{ Autonomia } \\
\hline FAST 1 & 100 & 56,72 & 1,0 & 0,14 & - \\
\hline FAST 2 & 100 & 63,37 & 1,0 & 0,14 & - \\
\hline FAST 3 & 100 & 75,58 & 1,0 & 0,12 & - \\
\hline FAST 4 & 100 & 70,22 & 1,0 & 0,13 & - \\
\hline \multicolumn{6}{|c|}{ Trabalho } \\
\hline FAST 5 & 100 & 73,92 & 1,0 & 0,12 & - \\
\hline FAST 6 & 97,37 & 70,22 & 0,91 & 0,13 & $0.81-1.00$ \\
\hline FAST 7 & 100 & 68,10 & 1,0 & 0,13 & - \\
\hline FAST 8 & 100 & 80,82 & 1,0 & 0,14 & - \\
\hline FAST 9 & 100 & 74,42 & 1,0 & 0,12 & - \\
\hline \multicolumn{6}{|c|}{ Cognição } \\
\hline FAST 10 & 97,37 & 54,43 & 0,94 & 0,14 & $0.85-1.00$ \\
\hline FAST 11 & 100 & 65,10 & 1,0 & 0,13 & - \\
\hline FAST 12 & 100 & 54,43 & 1,0 & 0,13 & - \\
\hline FAST 13 & 99,12 & 70,04 & 0,97 & 0,12 & $0.90-1.00$ \\
\hline FAST 14 & 96,49 & 66,57 & 0,89 & 0,13 & $0.76-0.97$ \\
\hline \multicolumn{6}{|c|}{ Finanças } \\
\hline FAST 15 & 98,68 & 55,06 & 0,97 & 0,13 & $0.90-1.00$ \\
\hline FAST 16 & 98,68 & 57,34 & 0,96 & 0,13 & $0.90-1.00$ \\
\hline \multicolumn{6}{|c|}{ Relações Interpessoais } \\
\hline FAST 17 & 100 & 67,96 & 1,0 & 0,13 & - \\
\hline FAST 18 & 96,49 & 58,91 & 0,91 & 0,12 & $0.83-0.96$ \\
\hline FAST 19 & 95,61 & 67,45 & 0,86 & 0,13 & $0.76-0.97$ \\
\hline FAST 20 & 96,49 & 63,07 & 0,90 & 0,13 & $0.76-0.97$ \\
\hline FAST 21 & 99,12 & 65,60 & 0,97 & 0,12 & $0.92-1.00$ \\
\hline FAST 22 & 100 & 63,67 & 1,0 & 0,12 & - \\
\hline \multicolumn{6}{|l|}{ Lazer } \\
\hline FAST 23 & 97,37 & 61,13 & 0,93 & 0,11 & $0.85-1.00$ \\
\hline FAST 24 & 93,86 & 60,02 & 0,84 & 0,12 & $0.72-0.93$ \\
\hline
\end{tabular}

a IC: intervalo de confiança. 
Tabela 3. Validade concorrente: correlações entre escore total e de subescalas da FAST com escore total da AGF

\begin{tabular}{lcc}
\hline FAST & $\begin{array}{c}\text { Correlaçöes com } \\
\text { escore total da AGF }\end{array}$ & p valores* \\
\hline Escore total & $-0,85$ & $<0,01$ \\
Domínio autonomia & $-0,87$ & $<0,01$ \\
Domíniotrabalho & $-0,77$ & $<0,01$ \\
Domínio cognição & $-0,86$ & $<0,01$ \\
Domínio eelações Interpessoais & $-0,85$ & $<0,01$ \\
Domínio finanças & $-0,88$ & $<0,01$ \\
Domínio lazer & $-0,77$ & $<0,01$ \\
\hline
\end{tabular}

Coeficiente de Spearman.

* Significativo até $5 \%$.

Tabela 4. Validade de cosntruto convergente: correlações entre escores das subescalas com escore total da FAST

\begin{tabular}{lcc}
\hline FAST & $\begin{array}{c}\text { Correlaçōes com } \\
\text { escore total (rho) }\end{array}$ & p valores* \\
\hline Domínio autonomia & 0,86 & $<0,01$ \\
Domínio trabalho & 0,84 & $<0,01$ \\
Domínio cognição & 0,93 & $<0,01$ \\
Domínio finanças & 0,88 & $<0,01$ \\
Domínio relações interpessoais & 0,94 & $<0,01$ \\
Domínio lazer & 0,85 & $<0,01$ \\
\hline${ }^{*}$ Coeficiente de Spearman. & & \\
${ }^{*}$ Significativo até5\%. & &
\end{tabular}

\section{Validade discriminativa}

Analisou-se a capacidade da FAST em discriminar os sujeitos em função da presença ou ausência de TDM e, também, entre as diferentes gravidades do transtorno (eutímicos/leves versus moderados/graves).

Para a avaliação da validade discriminativa entre o primeiro grupo (casos versus não casos), foram utilizados os dados da amostra total $(n=88)$ e aplicou-se a análise por meio da curva ROC. A área sob a curva encontrada foi de 99,5\%, com erro-padrão de 0,05 e intervalo de confiança de 95\% entre 0,98 e 1,0.

Calculou-se, também, a sensibilidade, especificidade, valor preditivo positivo, valor preditivo negativo, taxa de classificação correta e coeficiente de Youden (Sensibilidade + Especificidade - 1) para vários pontos de corte da FAST, visando identificar aquele mais apropriado para discriminar sujeitos depressivos dos controles ${ }^{28}$.

A nota de corte 10 foi aquela que melhor equilibrou os valores de sensibilidade (97,73\%), especificidade (100\%), valor preditivo positivo (100\%) e negativo (97,8\%), com taxa de classificação correta de $98,86 \%$ e coeficiente de Youden de $0,97^{28}$.

Para a avaliação da validade discriminativa entre o segundo grupo (saudáveis/depressivos eutímicos/leves versus depressivos moderados/graves), foram utilizados, também, os dados da amostra total $(n=88)$ e a área sob a curva encontrada foi de 99,97\%, com erro-padrão de 0,004 e intervalo de confiança de 95\% entre 0,99 e 1,0.

Para essa discriminação, a nota de corte mais apropriada foi a 32, com sensibilidade de 100\%, especificidade de $98,36 \%$, valor preditivo positivo de 96,4\%, valor preditivo negativo de 100\%, taxa de classificação correta de 98,86\% e coeficiente de Youden de 0,98.

\section{DISCUSSÃO}

Os prejuízos funcionais associados ao TDM, mesmo durante a fase de remissão clínica, têm sido documentados por vários estudos ${ }^{9,32-35}$. Dificuldades cognitivas, sono de baixa qualidade, lentificação e cansaço excessivo e repercussões negativas sobre os relacionamentos interpessoais e as habilidades sociais são alguns dos problemas mais comumente enfrentados pelos sujeitos depressivos e diretamente atrelados à queda da funcionalidade ${ }^{36-39}$. A despeito da relevância do tema, a maioria dos instrumentos disponíveis para aferição da funcionalidade é extensa e de difícil aplicabilidade, ou trata-se de escalas gerais que negligenciam aspectos importantes do funcionamento psicossocial| ${ }^{15,40,41}$.

Assim, o desenvolvimento de instrumentos de fácil aplicação e que contemplem as especificidades dos transtornos mentais é muito importante; nesse sentido, a escala FAST pode ser bastante apropriada.

Por meio da avaliação dos resultados obtidos, a FAST mostrou ser um instrumento válido e confiável para estimar o impacto da depressão sobre a funcionalidade em amostra brasileira de sujeitos com TDM.

A análise da consistência interna evidenciou alfa de Cronbach de 0,98 para a escala geral e, com exceção da subescala atividades de lazer, as demais subescalas apresentaram índices de, ao menos, 0,94, o que pode ser considerado muito bom, uma vez que, para o estudo de fidedignidade, se espera que o instrumento apresente índices maiores que 0,8029. Observou-se elevada estabilidade, com valor de Kappa ponderado maior que 0,84 [IC 95\% (0,72-0,93)]. Esses índices foram compatíveis com aqueles descritos por Rosa et al., com a versão original do instrumento ${ }^{18}$.

A validade concorrente foi estudada em relação a um instrumento de funcionalidade geral, a AGF, haja vista ser a mais utilizada e compartilhar com a FAST características importantes como ser multidimensional e heteroaplicada. As correlações encontradas foram bastante fortes, tanto para a escala FAST total como para cada subescala, evidenciando que cada um dos seis domínios da FAST (autonomia, trabalho, cognição, finanças, lazer e relações interpessoais) é bastante relevante e representativo da funcionalidade geral. No estudo original de validação em amostra de sujeitos bipolares, as correlações entre a FAST e a escala AGF evidenciaram valores 
menores que os do estudo em questão, mas ainda assim a correlação foi considerada forte $(r h o=-0,7, p<0,05)^{18}$.

Quanto à validade de construto convergente, observou-se forte correlação entre os escores de todas as subescalas com a escala total com coeficiente de Spearman > 0,84 ( $p<$ 0,05). Os domínios "cognitivo" e "relações interpessoais" foram os que apresentaram as maiores correlações (rho > 0,93, $\mathrm{p}<0,05)$. Esses achados são coerentes com a literatura e apontam que tanto os déficits cognitivos ${ }^{38,41-44}$ quanto o pobre funcionamento social ${ }^{45}$ são os que mais contribuem para os prejuízos associados à depressão, confirmando a validade de construto da escala.

Em relação à validade discriminativa, observou-se que a FAST foi capaz de discriminar "casos" de "não casos" e, também, depressivos moderados/graves dos demais. A nota de corte mais apropriada para diferenciar casos de não casos foi 10 (coeficiente de Youden de 0,97) e, para diferenciar depressivos moderados/graves dos demais, foi 32 (coeficiente de Youden de 0,98). A identificação de dois pontos de corte da escala reforça os achados prévios da literatura ${ }^{36-38}$ de que a funcionalidade é bastante representativa do TDM, sendo capaz de, por si só e com alto grau de significância, diferenciar casos de não casos e, também, distinguir as diferentes gravidades do TDM.

No cenário clínico, a caracterização da funcionalidade é, também, extremamente relevante, uma vez que a persistência de déficits funcionais, mesmo após remissão clínica, se associa a pior prognóstico ${ }^{8,9}$. E, nesse sentido, a escala FAST se mostrou eficiente ao demonstrar, com êxito, diferentes aspectos dos prejuízos funcionais, mesmo na ausência de sintomatologia importante, apontando para a grande utilidade da FAST para supervisão clínica e do processo de reabilitação de acometidos por TDM. Apesar do grande potencial de utilização da FAST evidenciado por suas boas propriedades psicométricas, é relevante assinalar algumas limitações. A primeira delas sustenta-se no fato de a amostra ser de conveniência e de ela não ter sido comparada ao perfil da população geral, impossibilitando sua generalização externa ${ }^{47}$. Por ser uma escala com 24 itens, o n amostral ideal para a avaliação psicométrica dessa escala deveria ser 240 sujeitos, no entanto apenas 88 foram avaliados. A despeito da amostra reduzida, a análise estatística foi bastante completa e os resultados encontrados foram muito bons. Contudo, essa diferença significativa faz com que este estudo tenha mais caráter exploratório, necessitando de estudos futuros com maiores amostras ${ }^{46}$.

Outra limitação deste estudo é que foram incluídos sujeitos com alto nível de escolaridade e bom suporte social. Essas características aumentam a probabilidade de que a amostra hipervalorize as dificuldades do domínio cognitivo e minimize os prejuízos do domínio autonomia. Alguma crítica deve ser feita à existência de dois domínios na escala (Finanças e Lazer) com menos de três itens ${ }^{48}$. Embora esse efeito possa ser minimizado pelo maior número de possibilidades de resposta por item (de zero a quatro), o não cumprimento desse pressuposto pode levar a problemas relacionados com a confiabilidade do construto ${ }^{47}$.

A falta de um instrumento "padrão-ouro" para aferir a funcionalidade em sujeitos com transtornos mentais é outra questão a ser apontada, uma vez que a validação concorrente foi realizada com a AGF, que, embora bastante utilizada, é uma escala geral e não dá conta das especificidades da FAST.

Finalmente, por ser um instrumento que se propõe a observar a efetividade do tratamento sobre a recuperação funcional em depressivos, a performance da FAST precisa ser testada em ensaios clínicos para se observar a aplicabilidade e suas propriedades psicométricas nesses contextos.

\section{CONCLUSÕES}

O ônus associado ao TDM é enorme, ocasionando elevados custos diretos e indiretos 5 . Pesquisas que se proponham a estudar o processo de reabilitação dos sujeitos com TDM são muito bem-vindas, embora ainda sejam escassas por carência de instrumentos específicos e validados para a aferição da funcionalidade nessa população.

A FAST apresentou excelentes propriedades psicométricas em amostra brasileira de sujeitos com TDM. O índice de persistência de sintomas residuais em TDM é alto e a escala FAST teria grande utilidade ao permitir dimensionar o impacto da depressão mesmo após a melhora clínica ou a remissão dos sintomas, potencializando o processo de reabilitação e minimizando o ônus associado a essa condição. A amostra reduzida faz com que este estudo tenha caráter exploratório, necessitando de estudos futuros com maiores amostras.

\section{CONTRIBUIÇÕES INDIVIDUAIS}

Juliana de Almeida Prado - Participou em todas as fases do estudo, desde a concepção e desenho, da coleta dos dados, da análise e interpretação dos resultados, e da elaboração do artigo e na aprovação de sua versão final.

Giovanni Gurgel Aciole - Colaborou com o delineamento do estudo, com a análise e interpretação dos dados, com a revisão crítica de seu conteúdo, com a elaboração do artigo e a aprovação de sua versão final.

Jair Lício Ferreira Santos - Participou em todas as fases do estudo, desde a concepção e desenho, da análise e interpretação dos resultados, da revisão crítica de seu conteúdo e da elaboração do artigo em suas versões inicial e a aprovação de sua versão final. 


\section{CONFLITOS DE INTERESSE}

Todos os autores declaram não possuir conflitos de interesse em relação a este estudo.

\section{AGRADECIMENTOS}

Aos responsáveis técnicos e aos pró-reitores de Gestão de Pessoas da Universidade Estadual Paulista Júlio de Mesquita Filho (Unesp) e da Universidade Federal de São Carlos (UFSCar), pelo apoio e confiança e por nos autorizarem a trabaIhar com a amostra de trabalhadores e pacientes dos serviços de saúde ocupacional e ambulatorial de ambas as instituições.

\section{REFERÊNCIAS}

1. Kessler RC, Bromet EJ. A epidemiologia da depressão entre culturas. Annu Rev Public Health 2013;34:119-38.

2. Santos ÉG, Siqueira MM. Prevalência dos transtornos mentais na população adulta brasileira: uma revisão sistemática de 1997 a 2009. J. Bras Psiquiatr. 2010;59(3):238-46.

3. Andrade LH, Wang YP, Andreoni S, Silveira CM, Alexandrino-Silva C, Siu ER, et al. Mental Disorders in Megacities: Findings from the São Paulo Megacity Mental Health Survey, Brazil. PLoS One. 2012;7(2):e31879

4. Bonadiman CSC, Passos VMA, Mooney M, Naghavi M, Melo APS. The burden of disease attributable to mental and substance use disorders in Brazil: Global Burden of Disease Study, 1990 and 2015. Rev Bras Epidemiol. 2017;20(Supl 1):191-204.

5. World Health Organization (WHO). Depression and other common mental disorders: global health estimates. Geneva: WH0; 2017. Disponível em: http://apps.who.int/iris/bitstream/ handle/10665/254610/WHO-MSD-MER-2017.2-eng.pdf. Acesso em: 20 dez. 2017.

6. American Psychiatric Association. Diagnostic and Statistical Manual of Mental Disorders, Fifth Edition (DSM-V). Arlington,VA: APA; 2013.

7. Moussavi S, Chatteriji S, VerdesE, Tandon A, Paterl V, Ustun B. Depression, chronic diseases and decrements in health: results from the World Health Surveys. Lancet. 2007;370(9590):851-8.

8. Mundt JC, Marks IM, Shear MK, Greist JH. The Work and Social Adjustment Scale: a simple measure of impairment in functioning. Br J Psychiatry. 2002;180:461-4.

9. Lerner D, Henke RM. What does research tell us about depression, job performance, and work productivity? J Occup Environ Med. 2008;50(4):401-10.

10. Gilmour H, Patten SB. Depression and work impairment. Health Rep. 2007;18(1):9-22.

11. Lawrence RE, Rasinski KA, Yoon JD, Meador KG, Koenig HG, Curlin FA. Primary care physicians' and psychiatrists' approaches to treating mild depression. Acta Psychiatr Scand 2012;126(5):385-92.

12. Wagner S, Doering B, Helmreich I, Lieb K, Tadic A. A meta-analysis of executive dysfunctions in unipolar major depression disorder without psychotic symptoms and their changes during antidepressant treatment. Acta Psychiatr Scand. 2012;125(4):281-92.

13. American Psychiatric Association (APA). Diagnostic and Statistical Manual of Menta Disorders. 4th ed (DSM-IV). Washington (DC): APA; 1994.

14. Organização Mundial da Saúde (OMS). Programa de Avaliação de Incapacidades WHO-DAS II: Manual de Treinamento: um guia para administração: Equipe de Classificação, Avaliação e Terminologia (CAT), Departamento de Medição e Sistemas de Informação em Saúde. 2004.

15. Berns S, Uzelac S, Gonzales C, Jaeger J. Methodological considerations of measuring disability in bipolar disorder: validity of the Multidimensional Scale of Independent Functioning. Bipolar Disord. 2007:9(1-2):3-10.

16. Gazalle FK, Frey BN, Hallal PC, Andreazza AC, Cunha AB, Santin A, et al. Mismatch between self-reported quality of life and functional assessment in acute mania: a matter of unawareness of illness? J Affect Disord. 2007;103(1-3):247-52.
17. Cacilhas AA, Magalhães PV, Ceresér KM, Wals JC, Weyne F, Rosa AR, et al. Validity of a short functioning test (FAST) in Brazilian outpatients with bipolar disorder. Value Health. 2009;12(4):624-7.

18. Rosa AR, Sánchez-Moreno J, Martínez-Aran A, Salamero M, Torrent C, Reinares M, et al. Validity and reliability of the Functioning Assessment Short Test (FAST) in bipolar disorder. Clin Pract Epidemiol Ment Health. 2007;3:5.

19. Swaine-Verdier A, Doward LC, Hagell P, Thorsen H, McKenna SP. Adapting quality of life instruments. Value Health. 2004;7(Suppl 1):S27-30.

20. Rothman ML, Beltran P, Cappelleri JC, Lipscomb J, TeschendorfB; Mayo/FDA Patient-Reported Outcomes Consensus Meeting Group. Patient-reported outcomes: conceptual issues. Value Health. 2007;10(Suppl 2):S66-75.

21. Amorim P. Mini International Neuropsychiatric Interview (MINI): validação de entrevista breve para diagnóstico de transtornos mentais. Rev. Bras. Psiquiatr. 2000;22(3):106-15.

22. Gallucci Neto J, Campos Júnior MS, Hübner CK. Escala de Depressão de Hamilton (HAM-D): revisão dos 40 anos de sua utilização. Rev Fac Ciênc Méd. 2001;3(1):10-4.

23. Bagby RM, Ryder AG, Schuller DR, Marshall MB. The Hamilton Depression Rating Scale: Has the Gold Standard Become a Lead Weight? Am J Psychiatry. 2004;16112():2163-77.

24. StataCorp. Stata Statistical Software: Release 13. College Station, TX: StataCorp, LP; 2013.

25. McDonald JH. Manual de Estatística Biológica. 3a ed. Baltimore, MD: Sparky House Publishing; 2014.

26. Siegel S. Estatística não paramétrica para as ciências do comportamento. São Paulo: McGraw-Hill do Brasil; 1975

27. Streiner DL, Norman GR. Health measurementscales: a practical guide to their development and use. 3th ed. London: Oxford University Press; 2003.

28. Youden W J, Steiner EH. Statistical Manual of the AOAC - Association of the Official Analytical Chemists. Washington, DC; 1975.

29. Nunnally J, Bernstein I. Psychometric Theory. 3rd ed. New York: McGraw-Hill; 1994.

30. Cohen JA. Coefficient of agreement for nominal scales. Educ Psychol Meas. 1960;20:37-46.

31. Landis JR, Koch GG. The measurement of observer agreement for categorical data. Biometrics. 1977;33(1):159-74.

32. Druss BG, Rosenheck RA, Sledge WH. Health and disability costs of depressive illness in a major US corporation. Am J Psychiatry. 2000;157(8):1274-8

33. Greenberg PE, Kessler RC, Birnbaum HG, Leong SA, Lowe SW, Berglund PA, et al. The economic burden of depression in the United States: how did it change between 1990 and 2000. J Clin Psychiatry. 2003;64(12):1465-75.

34. Stewart WF, Ricci JA, Chee E, Hahn SR, Morganstein D. Cost of lost productive work time among US workers with depression. JAMA. 2003;289(23):3135-44.

35. Adler DA, McLaughlin TJ, Rogers WH, Chang H, Lapitsky L, Lerner D. Job performance deficits due to depression. Am J Psychiatry. 2006;163(9):1569-76.

36. Austin MP, Mitchell P, Goodwin GM. Cognitive deficits in depression: possible implications for functional neuropathology. Br J Psychiatry. 2001;178(3):200-6.

37. Stordal Kl, Lundervold AJ, Egeland J, Mykletun A, Asbjørnsen A, Landrø NI, et al. Impairment across executive functions in recurrent major depression. Nord J Psychiatry. 2004;58(1):41-7.

38. Castaneda AE, Suvisaari J, Marttunen M, Perälä J, Saarni SI, Aalto-Setälä T, et al. Cognitive functioning in a population-based sample of young adults with a history of non-psychotic unipolar depressive disorders without psychiatric comorbidity. J Affect Disord. 2008;110(12):36-45.

39. Fried El, Nesse RM. The impact of individual depressive symptoms on impairment of psychosocial functioning. PloS One. 2014;9(2)

40. World Health Organization (WHO)). The International Classification of Diseases and Related Health Problems. 10th Revision. Geneva: WH0; 1992

41. Jaeger J, Berns SM, Czobor P. The multidimensional Scale of independent functioning: a new instrument for measuring functional disability in psychiatric populations. Schizofr Bull. 2003;29(1):153-67.

42. Kerr N, Scott J, Phillips M. Patterns of attentional deficits and emotional bias in bipolar and major depressive disorder. Br J Clin Psychol. 2005;44(3):343-56.

43. Mondal S, Sharma V, Das S, Goswani U, Gandhi A. Neurocognitive functioning patients of major depression. Indian J Physiol Pharmacol. 2007;51(1):69-75. 
44. Zimmerman M, McGlinchey JB, Posternak MA, Friedman M, Attiullah N, Boerescu D. How should remission from depression be defined? The depressed patient's perspective. Am J Psychiatry. 2006;163(1):148-50.

45. Porto P, Hermolin M, Ventura P. Alteraçōes neuropsicológicas associadas à depressão. Rev Bras Ter Comport Cogn. 2002;4(1):63-70.
46. Sousa VD, Rojijnassirat W. Translation, adaptation and validation of instruments or scales for use in cross-cultural health care research: a clear and user-friendly guideline. J Eval Clin Pract. 2011;17(2):268-74.

47. Hill M, Hill A. Investigação por questionário. 2a ed. Lisboa: Sílabo; 2009.

48. Mônaco J. Avaliação das qualidades psicométricas de uma escala. Lisboa: Manuscrito; 2009 